\title{
All the skin that's fit to print
}

The field of stretchable electronics has come a long way in recent years, with clever architectures and materials that make the devices more comfortable to wear and improve their performance. But making large-scale 'electronic skin'-soft, stretchable electronics that replicate skin functionalities-for people or robots has remained elusive. Writing in Nature, Zhenan Bao and colleagues ${ }^{1}$ report a system that enables scalable fabrication of intrinsically stretchable electronics with the highest device density achieved to date.

The challenge of generating flexible electronics has been tackled either by laying out rigid materials into stretchable designs or by seeking to use intrinsically stretchable building blocks. While materials that are not intrinsically stretchable can be mechanically designed into serpentine or wavy geometric layouts that confer stretchability, these approaches are constrained by the need to fit the components to the design. On the other hand, although some intrinsically stretchable polymers have good conductivity, they are sensitive to the solvents and ultraviolet light involved in commonly used fabrication methods. Functional electronic circuits from intrinsically stretchable materials have been very difficult to make and to scale into the sizes of electronics that would be needed for real applications.

Bao and colleagues ${ }^{1}$ develop a layer-bylayer method of deposition of intrinsically stretchable materials to make functional electronics. Instead of using transfer processes, which tend to have low yield and poor uniformity, the authors use a patterning approach. First, they use a silicon wafer that is coated with dextran, a water-soluble layer that ultimately enables release of the devices onto a stretchable substrate. Then they deposit a

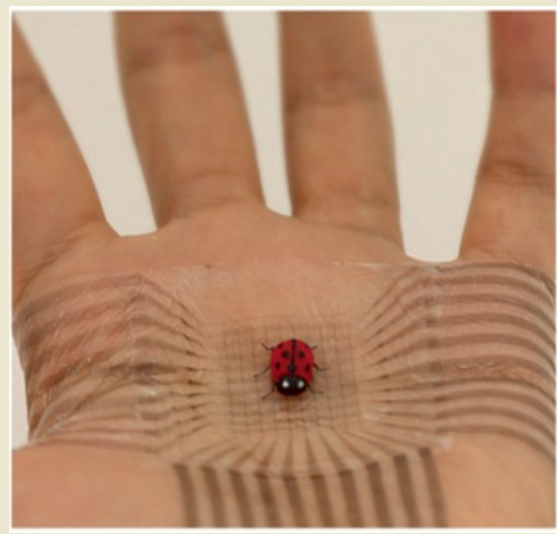

stretchable insulator, followed by sequential patterning of stretchable semiconductor and conductor layers. A layer of a stretchable substrate is laminated on, and the device is then released from the wafer. To complete the transistor structure, gate electrodes are deposited on the insulator.

The scientists build a 108-transistor arraywith an impressive density of 347 transistors per square centimeter-that incorporates the intrinsically stretchable semiconductor films they previously developed using nanoconfinement ${ }^{2}$. The transistor array is robust even when stretched to $100 \%$ strain 1,000 times in both directions and is stretchable up to $600 \%$ strain. It also has low-voltage operation and low power consumption, two additional features needed for compatibility with onskin applications.

"This electronic system can be compared to the active matrix that powers a flat-screen TV," says Martin Kaltenbrunner, associate professor at the Johannes Kepler University in Linz, Austria. "You have information in columns and rows, and in the back there is a transistor active matrix that powers it all. Having such a thing on a soft and stretchable form factor is really important for the field of electronic skin."

Bao and colleagues ${ }^{1}$ show that the process is scalable: they produce a large array of 6,300 transistors over an area of $4.4 \times 4.4 \mathrm{~cm}^{2}$. Although these dimensions are perhaps not quite as large as electronic skin would need to be, scaling up to this extent promises the ability to print much larger electronics using intrinsically stretchable materials. "Organic semiconductors are known to be printable, but going from the principles to developing a printable platform is a major breakthrough," says Kaltenbrunner. Printable systems may just be the answer to the problem of scale in stretchable electronics.

Integrating intrinsically stretchable tactile sensors into their circuits, Bao and colleagues ${ }^{1}$ demonstrate a transistor array that adheres comformably to a human hand and can detect the position of a six-legged artificial ladybug. Besides tactile sensing, human skin has other sensory nodes such as temperature, pressure and texture. Incorporating these multiple inputs, and potentially also self-healing features, with an intrinsically stretchable electronic circuit would result in a sensor that more closely resembles skin. Hybrid integration with silicon electronics for wireless data communication is another step we can expect in the future, says Kaltenbrunner. In the meantime, the ability to scale up stretchable electronics with printing intensifies the promise of skin-like sensing for humans and robots.

\section{Irene Jarchum, Senior Editor}

1. Wang, S. et al. Nature 555, 83-88 (2018). 2. Xu, J. et al. Science 355, 59-64 (2017).

\section{Research Highlights}

Papers from the literature selected by the Nature Biotechnology editors. (Follow us on Twitter, @NatureBiotech \#nbtHighlight)

Cell-of-origin patterns dominate the molecular classification of 10,000 tumors from 33 types of cancer Hoadley, K.A. et al. Cell 173, 291-304.e6 (2018)

Moving magnetoencephalography towards real-world applications with a wearable system

Boto, E. et al. Nature 555, 657-661 (2018)

iPSCs from a hibernator provide a platform for studying cold adaptation and its potential medical applications Ou, J. et al. Cell doi:10.1016/j.cell.2018.03.010 (2018)

Whole-organism clone tracing using single-cell sequencing

Alemany, A., Florescu, M., Baron, C.S., Peterson-Maduro, J. \& van Oudenaarden, A. Nature 556, 108-112 (2018)

The dTAG system for immediate and target-specific protein degradation

Nabet, B. et al. Nat. Chem. Biol. doi:10.1038/s41589-018-0021-8 (2018) 